\title{
Virological Non-Suppression and Associated Factors Among Adolescents and Youth Living with HIV in Ethiopia: A Facility-Based Case-Control Study
}

Yilkal Muchie Dires ( $\nabla$ yilkalmuchie@gmail.com )

All Africa TB Leprosy and rehabilitation training center (ALERT)

Tsegahun Manyazewal

All Africa TB Leprosy and rehabilitation training center (ALERT)

Hanlon Charlotte

All Africa TB Leprosy and rehabilitation training center (ALERT)

\section{Research}

Keywords: Virological non-suppression, HIV, depression, mental health, adolescents and youth, Ethiopia.

Posted Date: November 16th, 2021

DOl: https://doi.org/10.21203/rs.3.rs-1014693/v1

License: (c) (i) This work is licensed under a Creative Commons Attribution 4.0 International License.

Read Full License 


\section{Abstract}

Background: Virological non-suppression remains the major cause of treatment failure and mortality among adolescents and youth living with HIV in low- and middle-income countries (LMICs). Behavioral and mental health disorders affect adherence to antiretroviral therapy (ART) and the underlying virological suppression, but evidence from LMICs is relatively limited. We aimed to assess HIV virological non-suppression and associated factors among adolescents and youth attending ART in Ethiopia.

Methods: A 1:2 matched facility-based case-control study was conducted at ALERT Hospital, Addis Ababa, Ethiopia, among patients with HIVaged 12-24 years who were on ART and with a viral load > (case) or < (control) than 1000 copies/ml after at least six months on ART. The primary outcome was viral load non-suppression: a plasma viral load > 1000 copies/ $\mathrm{ml}$ based on two consecutive viral load measurements every three months. The primary exposure was depressive symptoms as measured using the PHQ-9 (Patient Health Questionnaire, 9-item version). Other variables include adherence to ART and sociodemographic characteristics of participants. Data were collected using structured, blinded lay interviewer-administered questionnaires, and data extraction forms. Conditional multivariable logistic regression was conducted using STATA version 14.2 to test the main hypotheses.

Results: The study enrolled 192 participants, with a median age of 18 years and $52.1 \%$ males. None of the participants reported smoking or chewing Khat (a leaf that stimulates mood). With the PHQ-9 tool, $47 \%, 28 \%$, and $25 \%$ had minimal or no, probable to mild and moderate to severe depression symptoms, respectively. Having depressive symptoms is associated with a higher conditional likelihood of virological failure (odds ratio $[\mathrm{OR}]=1.8, p<0.001$ ) and the association was stronger among those who have moderate or severe depressive symptoms (adjusted $\mathrm{OR}=5,95 \% \mathrm{Cl}$ [1.7-14.6]) and unadjusted $\mathrm{OR}=6.8$, $95 \% \mathrm{Cl}[3.1-14.8])$. Having moderate and severe depression is associated with non-adherence to ART (adjusted OR $=10.5$ ).

Conclusion: Non-adherence to ART medications, depressive symptoms, advanced WHO stage on ART initiation, poor social support and orphan status were independently associated with virological failure among HIV-infected adolescents and youths on ART. Having moderate and severe depression has higher odds of non-adherence and virological failure. We recommend integration and task-sharing of mental health and HIV care services to reduce virological failure in this group of people.

\section{Background}

HIV/AIDS remains a significant contributor to morbidity and mortality in adolescents and youth, especially in sub-Saharan Africa [1,2, and 33]. According to UNAIDS estimates, 71\% of the estimated 2.1 million adolescents and youth living with HIV (AYLHIV) in 2016 lived in nine sub-Saharan African countries and India [15]. Furthermore, $91 \%$ of adolescent deaths worldwide were reported in sub-Saharan Africa in 2016. In Ethiopia, HIV prevalence among adolescents and youth aged 10-24 years ranges from less than 0.1 percent in Somali to 1.3 percent in Gambella regional states, while the national figure stands 
at $0.2 \%$ (CSA and ICF, 2018). Although low, the absolute number of adolescents and young people affected by HIV is high given the Ethiopian population of over 100 million people. Ethiopia's progress towards the UNAIDS 90-90-90 target by 2020 report stands at 79-71-81 [33]. This number might even be overstated as different primary studies done in Ethiopia from primary to tertiary level care indicate a higher burden of viral non suppression, ranging from $22.6 \%$ to $26.3 \%[2,3,5$, and 14$]$.

The goal of ART is to restrict viral replication in CD4 cells. Achieving viral suppression protects people living with HIV from Acquired Immunodeficiency Syndrome (AIDS)-related illnesses, minimizes ART drug resistance and lowers the risk of transmission to others. Thus virological monitoring through plasma viral load (VL) quantification is an essential part of the monitoring of HIV patients undergoing antiretroviral therapy (ART). Routine VL monitoring allows timely detection of treatment failure, identification of patients in need of more intensive adherence support and protection against unnecessary switching to expensive and limited ART alternative regimen options.

Despite the introduction of the UNAIDS 90-90-90 target with universal testing and treatment to end the HIV epidemic in 2030, viral suppression outcomes remain a major challenge among adolescents and youth living with HIV in LMICs. According to a systematic review done in sub-Saharan countries, when stratified, PLHIV ages 15 to 24 years had lower median achievement in the treatment cascade (60-49-81), compared to PLHIV $\geq 25$ years (70-63-91) [15]. Studies have been carried out in a number of countries to investigate factors influencing adherence in these age groups. However, there are inconsistencies and disagreements among studies. In general, Factors that determine viral suppression outcomes are multidimensional and thus understanding the key barriers and outcomes of different factors can help to develop and standardize health service interventions that improve viral load suppression and improve health outcomes for adolescents and youth living with HIV. Factors other than sociodemographic and clinical factors might better explain differences in adherence and/or treatment failure in adolescents. This highlights the need for a more complex study that would look at the entire system which these patients are navigating as well as considering their behavioral and mental health. Of the known risk factors for viral non suppression, the contribution of mental health conditions has been less extensively investigated. While it is the most neglected condition in the HIV care package, according to a recent systematic review and meta-analysis, depression is highly prevalent among people living with HIV in Ethiopia $(35.8 \%)$ and the odds of non-adherence is high $(\mathrm{OR}=3.01)$ when co-morbid depression is present, Necho et al., 2021 [11].

Thus, this study aimed to assess virological non-suppression and associated factors among 12-24-yearold adolescents taking ART at ALERT Center, Addis Ababa, Ethiopia, 2021. The study is powered to detect the association of virological non-suppression and non-adherence to ART medications with the most neglected mental health condition, depression, using a hypothesis-driven case-control study. The hypothesis was that depressive symptoms have a significant association with virological nonsuppression and ART medication non-adherence.

\section{Methods}




\section{Study design and setting}

A1:2 matched case-control study was conducted among HIV-positive adolescents and youth aged 12-24 who were receiving ART at ALERT Center, Addis Ababa, Ethiopia. We do matching by age and sex to reduce bias and confounding at the design level.

The study was conducted in ALERT center Addis Ababa, Ethiopia. The institution is functioning as a training, research and medical service delivery center. More than 8, 000 people living with HIV are currently enrolled in the ART clinic at ALERT, of which more than 700 are children and youth aged below 24 years. This figure is the second-largest among hospitals that provide ART services in the city. ALERT serves as a referral hospital for the central, western, northwestern and southwestern parts of the city, together with the metropolitan area covering more than 5 million populations. The results obtained from this study may be extrapolated to Addis Ababa city administration and referral hospitals serving adolescent HIV care in the city.

\section{Participants}

The study population was HIV-positive adolescents and youth who were attending ART at the ALERT center. The eligibility criteria were as follows:

- Inclusion criteria

- 12 to 24-year-old adolescents on ART for at least six months and who were receiving follow-up at the ALERT center.

- Viral load $>1000$ copies/ml after at least six months on ART.

- Exclusion criteria

- No matched control is available.

- Refused or unable to give assent and/or consent.

- Presenting with an emergency medical condition.

- Unable to communicate with research staff (e.g. due to sensory problems, cognitive problems).

- Eligibility criteria for controls

- Adolescent/young person between 12 and 24 years of age.

- On antiretroviral therapy for at least six months

- Age matched to case (+/- 1 year).

- Sex-matched to case.

- Viral load $<1000$ copies/ml after six months of treatment.

Under 12 years children are excluded from the study as the depression screening tool is less sensitive to detect depression in under 12 years children. In addition consenting of parents was thought to be difficult 


\section{Recruitment}

Cases and controls were interviewed by two independent, trained general practitioners either on their appointment dates for ART follow-up or when they came for their support group meetings which were conducted on Saturdays every week. Those who did not belong to a support group and who did not come for review during the study period were interviewed by phone. Contact details were found in the VL tests registers. Eligible participants were approached by research staff to provide potential participants with information on the research and invite them to participate.

\section{Sample size calculation}

A recent systematic review and meta-analysis found a high burden of depression among people with HIV in Ethiopia (35.8\% pooled prevalence) and higher odds of non-adherence $(\mathrm{OR}=3.01)$ in depression compared to those without depression symptoms. Thus, the study is powered by this assumption to assess the association of depression with virological treatment failure in adolescents. The other factors were assessed as contributing factors for treatment failure. Using this study at $95 \%$ significance level ( $a=$ 0.05 ) and $80 \%$ power (delta $=0.8$ ), we assume a $35 \%$ exposure rate to depression among control groups and a 2.5 odds ratio of depression exposure in cases relative to the controls. Using STATA for 1:2 matched case-control, the number of cases required is 58 . Considering a $10 \%$ non-respondent rate, the ratio of cases to control will be 64 to 128 . Thus, the total sample size for the cases and the controls is 192.

\section{Outcome measures}

We used (1) fully structured, pre-tested, lay interviewer-administered questionnaires, and (2) data extraction forms. The questionnaires were designed in English language and translated into Amharic, the official language in Addis Ababa.

- Primary outcome: viral load suppression

- Viral non-suppression: elevated VL Ribo Nucleic Acid (RNA) copies of $>1000$ copies/ml in plasma in a person with HIV who has been on ART for at least six months. We took the most recent viral load as an outcome measurement.

- Primary exposure: depressive symptoms

- Depressive symptoms were measured using the Patient Health Questionnaire, 9-item version (PHQ-9) Kroenke et al, 2011]. Validity of the PHQ-9 tool in Ethiopia was investigated against the gold standard of the Mini International Neuropsychiatric Interview (MINI) diagnostic tool among 
cancer patients in Tikur Anbessa hospital, the national referral and teaching hospital, and showed a sensitivity of $88 \%$ and specificity of $78.1 \%$ to detect major depressive episode (MDE) [43]. Validity of the PHQ-9 for depression screening and the diagnosis was also done against psychiatrist administered semi-structure schedules for clinical assessment in neuropsychiatry (SCAN) interview among 926 Ethiopian adults in 2013 demonstrate good reliability and validity of the tool (sensitivity $=86 \%$ and specificity $=67 \%$ ), Bizu Gelaye et al. [44].

- Other explanatory variables: adherence to antiretroviral medication

- Adherence was objectively assessed using a physical pill count of dose units taken between the supply date and the review date. Good adherence was defined as adherence necessary to achieve full and durable viral suppression, thus taking ( $=$ or $>95 \%)$ of the pills. We operationalized the adherence level category as good, fair and poor for analysis as follows:

- Potential confounders: sociodemographic characteristics

- The demographic characteristics of the study participants were recorded, including age (number of completed years), marital status (married, single, divorced), gender (male or female), occupation (organization employed, self-employed, unemployed), alcohol intake (consumption of alcohol: daily, on weekends, holy days only, not at all), family income (amount in Birr per day), religion (Orthodox, Protestant, Muslim, others), living status (orphanage home, other caregivers, living with a partner, living with parents) orphan Status (double orphan, orphan mother, orphan father, not orphan), support group involvement (always involved, sometimes, rarely or never) and caregiver employment status (employed or not employed). Substance use including chewing khat, (never, sometimes, always), smoking (yes/no). All these data were obtained by face-to-face interviews. First viral load in the hospital (number of viral copies/ $\mu l$ ). Disclosure status (disclosed HIV status or not), WHO staging (WHO stage at ART initiation, if ART initiation was in other facilities the first WHO stage in the hospital will be taken), CD4 count (CD4 count at ART initiation), Drug S/E (Yes, No) chronic illness (having a chronic disease or not), Current ART regimen (first line second line or third line). These data were extracted from the participants' medical records and viral load records.

\section{Operational definitions}

- Good adherence: Drug adherence of $95 \%$ or $\leq 2$ missed drug doses of 30 doses or $\leq 3$ missed drug doses of 60 doses.

- Fair Adherence: Drug adherence of $85-94 \%$ or 3-5 missed drug doses of 30 doses or 3-9 missed drug doses of 60 doses.

- Poor Adherence: Drug adherence of $<85 \%$ or $\geq 6$ doses of missed ART drug doses of 30 doses or $>9$ doses missed ART drug doses of 60 doses. 
- Virological non-suppression: a plasma viral load above 1000 copies/ ml based on two consecutive viral load measurements after 3 months, with adherence support.

\section{Data collection}

Two independent general practitioners with experience in ART follow-up from adult ART (outside the pediatric ART care providers) were assigned for data collection. One of them collected data from the medical records while the other conducted participant interviews and administered the questionnaire. Data collectors were given adequate training on how to administer the questionnaires and how to complete the data extraction tools. Post-training knowledge was assessed using an interactive question and answer discussion on the second day. Pretesting of the questionnaire was performed by data collectors in a small sample of young people who were using ART and in the adult follow-up clinic. Findings were reviewed.

We used structured, pre-tested interviewer-administered questionnaires to collect data. The questionnaires were designed in English language then translated into Amharic language which is the official language in Addis Ababa. A review of the hospital medical records provided baseline pre-ART data before initiation of HIV treatment as well as current treatment and clinical status (see under confounder measurements section). The data collector who was assigned to conduct a face-to-face interview was not aware of whether the respondent is a case or control. To ensure this, the first person who was assigned to recruit eligible participants and to collect necessary data from medical records was also dedicated to remove VL reports and keep these separate until the interview had been completed. Each data entry item was double-checked independently by two data clerks and the entire data set was checked for consistency, and discrepancies were discussed and checked from the primary tool/questionnaire.

\section{Data analysis}

Data analysis was performed using STATA version 14.2 to generate frequencies of exposures, means, proportions and odds ratios. Bivariate analysis was conducted to assess associations between different exposures and virological failure. A p-value of $<0.05$ was deemed statistically significant in this analysis. Conditional multivariable logistic regression was conducted to test the main hypothesis of an association between depressive symptoms (primary exposure) and non-viral suppression (primary outcome) after adjusting for all pre-specified confounders.

\section{Ethical considerations}

Ethical approval to conduct the study was obtained from the Scientific and Ethics Review Committee of the Center for Innovative Drug Development and Therapeutic Trials for Africa (CDT-Africa), College of Health Sciences, Addis Ababa University, and the Institutional Review Board of the ALERT Center. Written 
informed consent was prepared using the American federal regulations consent/assent checklist. Consent was obtained from all study participants who were aged 18 years and above. For adolescents aged 12 to 18 years, assent was obtained. Confidentiality was assured and maintained through no use of names on questionnaires and keeping questionnaires under lock and key.

\section{Results}

\section{Overall characteristics}

We have approached 66 eligible cases. Of those eligible cases, 1 adolescent was not able to consent and was excluded from the study. One other adolescent didn't get an age match in the study period and was also excluded from the study. All approached eligible controls agreed to participate in the study and were able to consent and were enrolled.

\section{Socio-demographic characteristics}

Of the 192 study participants, $52.1 \%$ were males. The median age of participants was 18 years.

Regarding the educational status of the participants, 61 (31.8\%) and $102(53.1 \%)$ of participants were primary and secondary school students, respectively. Despite higher median age of 18 , only $19(9.9 \%)$ participants were currently college/university-level students and only 10 participants were college/university graduates. Double orphan status was $26.6 \%$ among cases and $9.4 \%$ among controls. Only $30 \%$ of cases lived with their parents. The mean family daily income in Birr was 116.349. Table 1 summarizes the sociodemographic and treatment-related information of study participants among cases and controls.

Table 1: Sociodemographic information 
Variables

Total Patients with

$(\mathrm{N}=192) \quad$ virological failure

$(\mathrm{N}=64)$

Mean age (years)

Sex

Male

Female

17.5

17.5

17.5

Participants without virological failure $(\mathrm{N}=128)$
100

$(52.1 \%)$

$34(53.1 \%)$

$30(46.9 \%)$

92
$(47.9 \%)$
$66(51.6 \%)$

$62(48.4)$

\begin{tabular}{lllll} 
Religion & Orthodox & $\begin{array}{l}125 \\
(65.1 \%)\end{array}$ & $48(75.0 \%)$ & $77(60.2 \%)$ \\
\hline Protestant & $\begin{array}{l}43(22.4 \\
\%)\end{array}$ & $13(20.3 \%)$ & $30(23.4 \%)$ \\
\hline Muslim & $\begin{array}{l}24(12.5 \\
\% 0\end{array}$ & $3(4.7 \%)$ & $21(16.4 \%)$
\end{tabular}

Employment

\begin{tabular}{|c|c|c|c|}
\hline $\begin{array}{l}\text { Self } \\
\text { employed }\end{array}$ & $7^{7(3.6 \%}$ & $3(4.7 \%)$ & $4(3.1 \%)$ \\
\hline Unemployed & $\begin{array}{l}172( \\
89.6 \%)\end{array}$ & 52 (81.3\%) & 120 (93.7\%) \\
\hline $\begin{array}{l}\text { Org. } \\
\text { employed }\end{array}$ & $\begin{array}{l}13( \\
6.8 \%)\end{array}$ & $9(14.1 \%)$ & $4(3.1 \%)$ \\
\hline
\end{tabular}

Orphan status

double

orphan

orphan

mother

orphan

father

not orphan
29

$(15.1 \%)$

$17(26.6 \%)$

$16(25.0 \%)$

$(17.2 \%)$

40

(20.8\%)

90

(46.9\%)
$12(9.4 \%)$

$17(13.3 \%)$

$29(22.6 \%)$

70 (54.7\%)

Living status

\begin{tabular}{llll}
$\begin{array}{l}\text { orphanage } \\
\text { home }\end{array}$ & $2(1 \%)$ & $1(1.6 \%)$ & $1(0.8 \%)$ \\
$\begin{array}{l}\text { other care } \\
\text { givers }\end{array}$ & $\begin{array}{l}31 \\
(16.1)\end{array}$ & $16(25.0 \%)$ & $15(11.7 \%)$ \\
\hline $\begin{array}{l}\text { living with } \\
\text { partner }\end{array}$ & $3(1.6 \%)$ & $2(3.1 \%)$ & $1(0.9 \%)$ \\
\hline
\end{tabular}

Page 9/22 


$\begin{array}{llll}\begin{array}{l}\text { living with } \\ \text { parents }\end{array} & 156 & 45(70.3 \%) & 111(86.7 \%)\end{array}$

\begin{tabular}{lllll}
$\begin{array}{l}\text { Care giver } \\
\text { employment }\end{array}$ & $\begin{array}{l}\text { Not } \\
\text { employed }\end{array}$ & $\begin{array}{l}35 \\
(18.2 \%)\end{array}$ & $18(28.1 \%)$ & $17(15 \%)$ \\
\cline { 2 - 5 } & Employed & $\begin{array}{l}157 \\
(81.8 \%)\end{array}$ & $46(71.9 \%)$ & $111(85 \%)$
\end{tabular}

\section{ART status}

The majority $(n=115)$ of participants among controls were taking first-line ART medications and 11 controls took second-line ART whereas 2 controls were taking unclassified regimen line. In comparison, 41 of the case participants were taking second-line ART medications (Table 2). However, comparison of results based on regimen lines was not possible due to a programmatic change of ART medications. Dolutegravir based regimen was newly introduced in the country as a first-line ART regimen in adults and adolescents and dolutegravir optimization was made among selected adolescents on second-line art regimen.

Table 2. Treatment status of study participants 
Variables

Total Variable Frequency among cases and controls

Variable Frequency among cases
Variable

Frequency among controls

\begin{tabular}{lllll}
$\begin{array}{l}\text { WHO Stage } \\
\text { during ART } \\
\text { initiation }\end{array}$ & stage 1 & $35(18.2 \%)$ & $1(1.6 \%)$ & $34(26.5 \%)$ \\
\cline { 2 - 5 } & stage 2 & $99(51.6 \%)$ & $37(57.8 \%)$ & $62(48.4 \%)$ \\
\cline { 2 - 5 } & stage 3 & $50(26.0 \%)$ & $21(32.8 \%)$ & $29(22.6 \%)$ \\
\cline { 2 - 5 } & stage 4 & $8(4.2 \%)$ & $5(7.8 \%)$ & $3(2.3 \%)$ \\
\hline $\begin{array}{l}\text { Previous history } \\
\text { of TB treatment }\end{array}$ & Yes & $49(25.5 \%)$ & $26(40.6 \%)$ & $23(17.9 \%)$ \\
\cline { 2 - 6 } & No & $143(74.5 \%)$ & $38(59.4 \%)$ & $105(82.0 \%)$
\end{tabular}

Current ART

regimen line

\begin{tabular}{|c|c|c|c|}
\hline first line & 135 (70.3\%) & $20(31.3 \%)$ & 115 (89.8\%) \\
\hline second line & $52(27.1 \%)$ & 41 (64.1\%) & $(8.6 \%)$ \\
\hline third line & $1(0.5 \%)$ & $1(1.6 \%)$ & $\mathrm{O}(0 \%)$ \\
\hline Unclassified & $4(2.1 \%)$ & $2(3.6 \%)$ & $2(1.5 \%)$ \\
\hline
\end{tabular}

All controls had undetectable current viral load whereas the mean current viral load of cases was 36,107 . The mean duration of ART use among cases and controls was not significantly different and the overall mean duration of ART use was 135 months. The baseline viral load had not been determined for any participant, but the mean baseline CD 4 count was 490 , with no significant difference between cases and controls (Figure 1). The mean duration of time since the last/most recent viral load was determined was 111 days. None of the adolescents had recent CD4 counts, with treatment response largely followed by viral load determination every six months. $40 \%$ of the case and $18 \%$ of controls have a previous history of TB treatment. HIV status had been disclosed to all adolescents and youths.

\section{Depression}

Two-way tabulation using fisher's exact test shows significantly different depressive symptoms among cases and controls ( $p<0.001$. Table 3 shows the PHQ-9 depression screening tool symptom score frequency and severity among cases and controls.

Table 3: depression burden among cases and controls 


\begin{tabular}{lccllll}
\hline Viral load category & No & Minimal & Mild & Moderate & Moderately severe (15-19) & Severe \\
& $(0)$ & $(1-4)$ & $(5-9)$ & $(10-14)$ & & $(>19)$ \\
\hline Control & 52 & 18 & 43 & 7 & 8 & 0 \\
\hline Case & 11 & 10 & 11 & 12 & 19 & 1 \\
\hline Total & 63 & 28 & 54 & 19 & 27 & 1
\end{tabular}

\section{Depression and adherence to ART}

We were also able to notice a higher rate of poor adherence to ART medications among moderate and severely depressed children. Ten out of 11 participants who had poor ART medication adherence had a concomitant moderate to moderately severe depression (Table 4).

Table 4: Two-way cross-tabulation of depression and ART medication adherence status

\begin{tabular}{llllll} 
PHQ-9 depression screening score & \multicolumn{5}{c}{ ART adherence level category } \\
\cline { 2 - 5 } & Good & Fair & Poor & Total \\
\hline Mild, minimal or no depression & 126 & 18 & 11 & 145 \\
& & & & \\
\hline Moderate to severe depression & 14 & 23 & 10 & 47 \\
\hline Total & & & & \\
\hline
\end{tabular}

We summarized the means and frequencies of PHQ-9 depression screening scores among cases and controls in association with the level of ART adherence. Cases who experienced poor ART adherence had a more advanced depression with, a mean depression score greater than 15 which is moderately severe depression (Table 5).

Table 5: Association between depression scores and ART adherence among cases and controls 
ART adherence level category

\begin{tabular}{lllll}
\cline { 3 - 5 } Variable type & Good & Fair & Poor & Total \\
Controls & & & & \\
Mean & 3.53 & 11.5 & 0 & 4.6 \\
Frequency & 111 & 17 & - & 128 \\
\hline
\end{tabular}

Cases

$\begin{array}{lllll}\text { Mean } & 7.6 & 8.5 & 15.45 & 9.23 \\ \text { Frequency } & 29 & 24 & 11 & 64\end{array}$

Total

$\begin{array}{lllll}\text { Mean } & 4.4 & 9.7 & 15.45 & 6.14 \\ \text { Frequency } & 140 & 41 & 11 & 192\end{array}$

\section{Factors associated with virological non-suppression}

We perform conditional logistic regression using STATA version 14.2. On univariate and multivariate conditional logistic regression analysis; orphan status, living status, ART medication adherence, less frequent involvement to monthly adolescent and youth support group, advanced WHO stage during art initiation and depressive symptoms were associated with higher odds of virological failure (Table 6). The odds of having virological failure with depressive symptoms was higher when we condition them to minimal and mild depression in one category and moderate and severe depression in another categorical level.

Table 6: A Univariate and Multivariate Conditional (fixed-effects) logistic regression modeling to determine the association of depression and virological failure $(n=192$. 
Virological failure

Univariate analysis

Multivariate analysis

$\left.\begin{array}{lllll}\text { OR } & P>Z & 95 \% & \text { OR } & P>Z\end{array}\right]$

$\mathrm{Cl}$

$\mathrm{Cl}]$

\begin{tabular}{|c|c|c|c|c|c|c|}
\hline $\begin{array}{l}\text { Depression category level as minimal to mild } \\
\text { versus moderate to severe depression }\end{array}$ & 6.8 & 0.000 & $\begin{array}{l}{[3.1-} \\
14.8]\end{array}$ & 4.974 & 0.004 & $\begin{array}{l}{[1.7-} \\
14.6]\end{array}$ \\
\hline Adherence level as good, fair, and poor & 8.0 & 0.000 & $\begin{array}{l}{[3.5-} \\
18.4]\end{array}$ & 10.90 & 0.000 & $\begin{array}{l}{[2.4-} \\
21.9]\end{array}$ \\
\hline Support group involvement & 6.5 & 0.000 & $\begin{array}{l}{[2.4-} \\
17.7]\end{array}$ & 7.23 & 0.002 & $\begin{array}{l}{[2.4-} \\
48.9]\end{array}$ \\
\hline WHO stage during ART initiation & 2.3 & 0.000 & $\begin{array}{l}{[1.5-} \\
3.5]\end{array}$ & 3.11 & 0.001 & $\begin{array}{l}{[1.6-} \\
6.2]\end{array}$ \\
\hline
\end{tabular}

NB: OR- ods ration; WHO- World Health Organization; Prob > Chi2 $=0.0000$

Variables that demonstrate significant association in the univariate conditional regression analysis were adjusted for the multivariate conditional regression analysis of the respective variables. Depressive symptoms were significantly associated with higher odds of ART medication adherence both on the univariate and multivariate logistic regression (crude odds ratio $=11.56$, adjusted odds ratio $=10.5, p=$ 0.0001). Tables 6 and 7 summarizes the univariate and multivariate output results with the respective odds ratio and confidence interval. Though significant association was found between support group involvement and WHO stage during ART initiation to ART adherence, multivariable conditional regression adjusted for depression did not indicate association.

Table 7: Univariate and multivariate logistic regression to determine the association between adherence to ART medications and other independent variables

Univariate conditional logistic regression

\begin{tabular}{lllll} 
Adherence level & $\mathrm{OR}$ & $\mathrm{Z}$ & $\mathrm{P}>\mathrm{Z}$ & {$[95 \% \mathrm{Cl}]$} \\
\hline Depression category & 11.6 & 4.53 & 0.000 & {$[4.0-33]$} \\
\hline Support group involvement & 3.2 & 2.13 & 0.033 & {$[1.1-9.5]$} \\
\hline WHO stage during ART initiation & 1.9 & 2.30 & 0.021 & {$[1.1-3.3]$}
\end{tabular}

Multivariate conditional logistic regression

\begin{tabular}{lllll}
\hline Depression category & 10.5 & 4.23 & 0.000 & {$[3.5-31]$} \\
\hline Support group involvement & 2.6 & 1.31 & 0.189 & {$[0.6-11]$} \\
\hline WHO stage during ART initiation & 1.6 & 1.14 & 0.254 & {$[0.7-3.5]$}
\end{tabular}




\section{Discussion}

In this study, we assess HIV virological non-suppression and associated factors among adolescents and youth attending ART in Ethiopia. We found that having depressive symptoms is associated with a higher conditional likelihood of both viral non-suppression and non-adherence to ART medications. The association was stronger among those adolescents who have moderate or severe depressive symptoms $(A O R=5$, and Crude $O R=6.8$ ). The findings in our study are supported by several other studies. Psychosocial interventions demonstrate small to moderate effect size on ART medication adherence, Pascalle Spaan et al, 2018 [12]. According to data from HIV-infected persons enrolled at a cohort study in Washington DC between January 2011 and June 2014, those with diagnoses of mental health issues or depression have a lower likelihood of sustained viral suppression. This is in agreement with a previous systematic review from 29 studies that included a total of 12,243 participants (Ref).

Data synthesized from these studies revealed that depression treatments and mental health interventions are effective for enhancing adherence to antiretroviral regimens. The odds of a person adhering to ART are $83 \%$ better if the patient is treated for depression, and the risk of no adherence is $35 \%$ greater among those who do not receive depression treatment. Studies in which participants were diagnosed with clinical depression or had, on average, moderate-to-severe depressive symptoms demonstrated larger effects than studies in which participants had mild depressive symptoms [9]. One study in Ethiopia performed a detailed systematic review to assess the prevalence of depression and the associated factors for the co-occurrence of depression in people living with HIV [11]. Our finding is however is significantly larger with depression prevalence 59.35 and $82 \%$ among controls and cases. This difference might be due to study population difference as our study targets only adolescents and youth aged 12-24 years. The figure is also significantly larger than the expected burden in the general population which stands at $17 \%$. This difference might be explained by the most commonly reported additional risk factors among HIV positive patients, according to the systematic review mentioned above, Mogessie Necho, Ethiopia, 2018, presence of perceived HIV stigma, poor social support, poor medication adherence, opportunistic infection and advanced stage of AIDS are most commonly identified risks to depression. The pooled adjusted odds ratio (AOR) of perceived HIV stigma among the indicated studies was 3.75. Six studies reported poor social support as an associated factor for depression in HIV patients and the pooled AOR was found to be 6.22. Moreover, the average odds ratio of poor medication adherence, presence of opportunistic infection, and advanced stages of AIDS were 3.03,5.5, and 5.43 respectively. Despite this high burden of depression among HIV clients, psychiatric disorders are neither well addressed nor studied in Ethiopia HIV care.

We have assessed possible confounding variables and sociodemographic variables. Of the assessed sociodemographic variables, orphan status and living status were associated significantly with virological failure. Being a double orphan was associated with higher odds of virological failure compared to single orphan or non-orphan (crude $\mathrm{OR}=1.5, \mathrm{P}<0.001$ ). There was also a slightly higher 
association between living status and virological failure (crude $O R=0.6, p<0.01$. Having a caregiver was associated with a lower conditional likelihood of virological failure and non-adherence (crude OR = 1.6).

This is in agreement with other studies done in Ethiopia and other countries. According to Zvanaka Sithole et al. 2017, Zambia, of the socio-demographic factors assessed, only two of the variables impacted the outcome. Those who had treatment buddies were 0.6 times less likely to have virological failure compared to those who did not (13). Another study on the importance of caregivers in the outcome of pediatric HIV management in Kenya found that treatment failure was associated with not having both parents as caregivers. In Ethiopia, several studies evaluate the association of having caregivers and caregivers being HIV serology positive with viral suppression. Those who had HIV-positive caregivers were 0.5 times less likely to develop Virological failure as compared to those who did not [1, 2, $5,13]$. However, in our study, we do not assess the HIV status of caregivers and families due to ethical reasons.

We also assessed behavioral risk factors including adherence to ART medications, smoking, and alcohol use and chewing khat. However, none of the adolescents/youths smoke or chew khat. Alcohol use rate is also low among adolescents and there is no significant difference among groups. Nonadherence to ART medications as a risk behavior to ART treatment outcome has a strong association with Virological failure on both univariate and multivariate conditional logistic regression (crude $\mathrm{OR}=7.97$, adOR=10.95, $p<0.0001)$. This in agreement with recent study in Ethiopia, poor adherence to ART increased Virological failure by higher odds $(A O R=16.09)[2]$, with similar country-specific findings in Africa (Zimbabwe $n=102,18.5$; SA $n=134,14.5$, Zambia $n=256,18.15)[1,2,13,14,22]$. The good adherence rate, in our study, among the total participants and cases, was $72 \%$ and $45 \%$, respectively. This is in agreement with another study by Jeremy L Ross 2019, a prospective cohort study among 250 HIV infected and 59 matched HIV uninfected adolescents in Malaysia, Thailand, and Vietnam from July 2013March 2017, which found $60 \%$ self-reported adherence $\geq 95 \%$ at week 144 [8].

Despite the higher odds of Virological failure among non-adherent adolescents, 29 (45\%) and 41 (64\%) of cases good have good and fair adherence to ART medications respectively. Thus our study found a high burden of virological non suppression among sufficiently adhered adolescents, $45 \%$ of all adolescents who did not achieve viral suppression were sufficiently adhered [10]. This clearly indicates the presence of other independent risk factors for viral suppression regardless of adherence status, like depression. Our finding is also supported by several other studies as mentioned in the literature review part. This may also be an important indicator and notification for the necessity in developing a highly sensitive and specific approach to adherence measurement in adolescents and youth. Despite this fact, strict adherence monitoring using available methods is the single most important method of measurement for Virological failure and/or treatment outcome. This study has a limitation in that it was conducted in a single referral hospital and the generalizability of the results could be affected. The study has strengths in that it indicated the association of an important and less understood mental health condition, 
depression, and viral suppression among adolescents and youth living with HIV using a hypothesis-driven matched case-control method.

\section{Conclusion}

According to the results of our study, non-adherence to ART medications, depressive symptoms, advanced WHO stage on ART initiation and not involving in weekly support group meetings were independently associated with virological failure among adolescents and youths taking ART. The study also found a higher rate of non-adherence among adolescents affected by depression. Having moderate and severe depression has higher odds of non-adherence and virological failure. We recommend integration and task-sharing of mental health and HIV care services to reduce virological failure in this group of people.

\section{Declarations}

\section{Funding}

This study was supported by the Center for Innovative Drug Development and Therapeutic Trials for Africa (CDT-Africa), College of Health Sciences, Addis Ababa University, Ethiopia.

\section{Ethics approval and consent to participate}

Ethical approval to conduct the study was obtained from the Scientific and Ethics Review Committee of the Center for Innovative Drug Development and Therapeutic Trials for Africa (CDT-Africa), College of Health Sciences, Addis Ababa University, and the Institutional Review Board of the ALERT Center. For adolescents aged 12 to 18 years, assent was obtained. Confidentiality was assured and maintained through no use of names on questionnaires and keeping questionnaires under lock and key.

\section{Authors' contributions}

Study conception, data acquisition, synthesis, and first draft: YM. Data acquisition and synthesis, review the draft: $\mathrm{CH}, \mathrm{TM}$. All authors reviewed and approved the final version for publication.

\section{Acknowledgements}

The authors thank participant adolescents and youths in the study for their contributions to the body of knowledge.

\section{Competing interests}

The authors have declared that no competing interests exist.

\section{Consent for publication}


Participants provided informed consent to publish the identified data.

\section{Data availability}

Data used for all analyses are included in the manuscript.

\section{Authors' information}

All Africa TB Leprosy and rehabilitation training (ALERT) center, Addis Ababa, Ethiopia, Center for Innovative Drug Development and Therapeutic Trials for Africa (CDT-Africa), College of Health Sciences, Addis Ababa University, Addis Ababa, Ethiopia

Dr. Yilkal Muchie

Center for Innovative Drug Development and Therapeutic Trials for Africa (CDT-Africa), College of Health Sciences, Addis Ababa University, Addis Ababa, Ethiopia

Dr. Tsegahun Manyazewal

Health Service and Population Research Department, Centre for Global Mental Health, Institute of Psychiatry, Psychology and Neuroscience, King's College London, London, UK, Department of Psychiatry, School of Medicine, College of Health Sciences, Addis Ababa University, Addis Ababa, Ethiopia.

Dr. Hanlon Chalotte

Address correspondence: Dr. Yilkal Muchie Dires. yilkalmuchie@gmail.com. Center for Innovative Drug Development and Therapeutic Trials for Africa (CDT-Africa), College of Health Sciences, Addis Ababa University, P.O. Box 9086, Addis Ababa, Ethiopia

\section{References}

1. Emagnu A, Abay Z, Bulti AB, Animut Y. Determinants of Virologic Failure among Adult HIV Patients on First-Line Antiretroviral Therapy at Waghimra Zone, Northern Ethiopia: A Case-Control Study. Adv Public Heal. Published online 2020. doi:10.1155/2020/1929436

2. Haile GS, Berha AB. Predictors of treatment failure, time to switch and reasons for switching to second line antiretroviral therapy in HIV infected children receiving first line anti-retroviral therapy at a Tertiary Care Hospital in Ethiopia. BMC Pediatr. Published online 2019. doi:10.1186/s12887-0191402-1

3. Desta AA, Woldearegay TW, Futwi N, et al. HIV virological non-suppression and factors associated with non-suppression among adolescents and adults on antiretroviral therapy in northern Ethiopia: A retrospective study. BMC Infect Dis. Published online 2020. doi:10.1186/s12879-019-4732-6

4. Mgelea EM, Kisenge R, Aboud S. Detecting virological failure in HIV-infected Tanzanian children. South African Med J. 2014;104(10):696-699. doi:10.7196/SAMJ.7807 
5. Reif LK, Abrams EJ, Arpadi S, et al. Interventions to Improve Antiretroviral Therapy Adherence Among Adolescents and Youth in Low- and Middle-Income Countries: A Systematic Review 2015-2019. AIDS Behav. Published online 2020. doi:10.1007/s10461-020-02822-4

6. Ross JL, Teeraananchai S, Lumbiganon P, et al. A Longitudinal Study of Behavioral Risk, Adherence, and Virologic Control in Adolescents Living with HIV in Asia. J Acquir Immune Defic Syndr. Published online 2019. doi:10.1097/QAl.0000000000002008

7. Zgambo M, Kalembo FW, Mbakaya BC. Risky behaviours and their correlates among adolescents living with HIV in sub-Saharan Africa: A systematic review. Reprod Health. Published online 2018. doi:10.1186/s12978-018-0614-4

8. Jones HS, Floyd S, Stangl A, et al. Association between HIV stigma and antiretroviral therapy adherence among adults living with HIV: baseline findings from the HPTN 071 (PopART) trial in Zambia and South Africa. Trop Med Int Heal. Published online 2020. doi:10.1111/tmi.13473

9. Sin NL, DiMatteo MR. Depression treatment enhances adherence to antiretroviral therapy: A metaanalysis. Ann Behav Med. Published online 2014. doi:10.1007/s12160-013-9559-6

10. Natukunda J, Kirabira P, Ong KIC, Shibanuma A, Jimba M. Virologic failure in HIV-positive adolescents with perfect adherence in Uganda: a cross-sectional study. Trop Med Health. Published online 2019. doi:10.1186/s41182-019-0135-z

11. Necho M, Belete A, Tsehay M. Depressive symptoms and their determinants in patients who are on antiretroviral therapy in the case of a low-income country, Ethiopia: a systematic review and metaanalysis. Int J Ment Health Syst. Published online 2021. doi:10.1186/s13033-020-00430-2

12. Spaan P, van Luenen S, Garnefski N, Kraaij V. Psychosocial interventions enhance HIV medication adherence: A systematic review and meta-analysis. J Health Psychol. Published online 2020. doi:10.1177/1359105318755545

13. Sithole Z, Mbizvo E, Chonzi P, et al. Virological failure among adolescents on ART, Harare City, 2017a case-control study. BMC Infect Dis. Published online 2018. doi:10.1186/s12879-018-3372-6

14. Sibhat M, Kassa M, Gebrehiwot $H_{\text {. }}<p>$ Incidence and Predictors of Treatment Failure Among Children Receiving First-Line Antiretroviral Treatment in General Hospitals of Two Zones, Tigray, Ethiopia, 2019</p>. Pediatr Heal Med Ther. Published online 2020. doi:10.2147/phmt.s243656

15. Green D, Tordoff DM, Kharono B, et al. Evidence of sociodemographic heterogeneity across the HIV treatment cascade and progress towards 90-90-90 in sub-Saharan Africa - a systematic review and meta-analysis. J Int AIDS Soc. Published online 2020. doi:10.1002/jia2.25470

16. Ojha CR, Shakya G, Dumre SP. Virological and Immunological Status of the People Living with HIV/AIDS Undergoing ART Treatment in Nepal. Biomed Res Int. 2016;2016(July). doi:10.1155/2016/6817325

17. Abah IO, Ncube NBQ, Bradley HA, AgbaJi OO, Kanki P. Antiretroviral Therapy-associated Adverse Drug Reactions and their Effects on Virologic Failure- A Retrospective Cohort Study in Nigeria. Curr HIV Res. Published online 2019. doi:10.2174/1389450120666190214144609 
18. Teeraananchai S, Kerr SJ, Gandhi M, et al. Determinants of Viral Resuppression or Persistent Virologic Failure After Initial Failure With Second-Line Antiretroviral Treatment Among Asian Children and Adolescents With HIV. J Pediatric Infect Dis Soc. Published online 2020.

doi:10.1093/jpids/piz034

19. Mziray SR, Kumburu HH, Assey HB, et al. Patterns of acquired HIV-1 drug resistance mutations and predictors of virological failure in Moshi, Northern Tanzania. PLoS One. Published online 2020. doi:10.1371/journal.pone.0232649

20. Miti S, Handema R, Mulenga L, et al. Prevalence and characteristics of HIV drug resistance among antiretroviral treatment (ART) experienced adolescents and young adults living with HIV in Ndola, Zambia. PLoS One. 2020;15(8 August):1-15. doi:10.1371/journal.pone.0236156

21. Bircher RE, Ntamatungiro AJ, Glass TR, et al. High failure rates of protease inhibitor-based antiretroviral treatment in rural Tanzania - A prospective cohort study. PLoS One. Published online 2020. doi:10.1371/journal.pone.0227600

22. Nega J, Taye S, Million Y, Rodrigo C, Eshetie S. Antiretroviral treatment failure and associated factors among HIV patients on first-line antiretroviral treatment in Sekota, northeast Ethiopia. AIDS Res Ther. Published online 2020. doi:10.1186/s12981-020-00294-z

23. Chhim K, Mburu G, Tuot S, et al. Factors associated with viral non-suppression among adolescents living with HIV in Cambodia: A cross-sectional study. AIDS Res Ther. Published online 2018. doi:10.1186/s12981-018-0205-z

24. Umar E, Levy JA, Bailey RC, Donenberg G, Hershow RC, Mackesy-Amiti ME. Virological Nonsuppression and Its Correlates Among Adolescents and Young People Living with HIV in Southern Malawi. AIDS Behav. Published online 2019. doi:10.1007/s10461-018-2255-6

25. Telele NF, Kalu AW, Marrone G, et al. Baseline predictors of antiretroviral treatment failure and lost to follow up in a multicenter countrywide HIV-1 cohort study in Ethiopia. PLoS One. Published online 2018. doi:10.1371/journal.pone.0200505

26. Mgelea EM, Kisenge R, Aboud S. Detecting virological failure in HIV-infected Tanzanian children. South African Med J. 2014;104(10):696-699. doi:10.7196/SAMJ.7807

27. Martelli G, Antonucci R, Mukurasi A, Zepherine H, Nöstlinger C. Adherence to antiretroviral treatment among children and adolescents in Tanzania: Comparison between pill count and viral load outcomes in a rural context of Mwanza region. PLoS One. Published online 2019. doi:10.1371/journal.pone.0214014

28. Nega J, Taye S, Million Y, Rodrigo C, Eshetie S. Antiretroviral treatment failure and associated factors among HIV patients on first-line antiretroviral treatment in Sekota, northeast Ethiopia. AIDS Res Ther. Published online 2020. doi:10.1186/s12981-020-00294-z

29. Xu L, Munir K, Kanabkaew C, Le Coeur S. Factors influencing antiretroviral treatment suboptimal adherence among perinatally hivinfected adolescents in Thailand. PLoS One. Published online 2017. doi:10.1371/journal.pone.0172392 
30. Thior I, Rowley E, Mavhu W, et al. Urban-rural disparity in sociodemographic characteristics and sexual behaviors of HIVpositive adolescent girls and young women and their perspectives on their male sexual partners: A cross-sectional study in Zimbabwe. PLoS One. Published online 2020. doi:10.1371/journal.pone.0230823

31. Mabunda K, Ngamasana EL, Babalola JO, Zunza M, Nyasulu P. Determinants of poor adherence to antiretroviral treatment using a combined effect of age and education among human immunodeficiency virus infected young adults attending care at letaba hospital hiv clinic, Limpopo Province, South Africa. Pan Afr Med J. 2019;32:1-14. doi:10.11604/pamj.2019.32.37.17722

32. Federal HIV/AIDS Prevention and Control Office. HIV Prevention in Ethiopia: National Road Map. 2018;(November 2018):1-43.

33. Bekker LG, Montaner J, Ramos C, et al. IAPAC guidelines for optimizing the HIV care continuum for adults and adolescents. J Int Assoc Provid AIDS Care. 2015;14(Supplement 1):S3-S34. doi:10.1177/2325957415613442

34. Nigusso FT, Mavhandu-Mudzusi AH. Magnitude of non-adherence to antiretroviral therapy and associated factors among adult people living with HIV/AIDS in Benishangul-Gumuz Regional State, Ethiopia. PeerJ. 2020;8. doi:10.7717/peerj.8558

35. Burns R, Magalasi D, Blasco P, et al. "We give them threatening advice...": expectations of adherence to antiretroviral therapy and their consequences among adolescents living with HIV in rural Malawi. $J$ Int AIDS Soc. 2020;23(3). doi:10.1002/jia2.25459

36. Mellins $\mathrm{CA}$, Malee KM. Understanding the mental health of youth living with perinatal HIV infection: Lessons learned and current challenges. J Int AIDS Soc. 2013;16(Lmic). doi:10.7448/IAS.16.1.18593

37. Wood SM, Lowenthal E, Lee S, Ratcliffe SJ, Dowshen N. Longitudinal Viral Suppression among a Cohort of Adolescents and Young Adults with Behaviorally Acquired Human Immunodeficiency Virus. AIDS Patient Care STDS. Published online 2017. doi:10.1089/apc.2017.0078

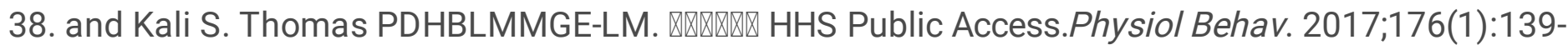
148. doi:10.1007/s10461-017-1915-2. Tobacco use and sustained viral suppression in youth living with HIV

39. Adejumo OA, Malee KM, Ryscavage P, Hunter SJ, Taiwo BO. Contemporary issues on the epidemiology and antiretroviral adherence of HIV-infected adolescents in sub-Saharan Africa: A narrative review. J Int AIDS Soc. 2015;18(1). doi:10.7448/IAS.18.1.20049

40. Fokam J, Billong SC, Jogue F, et al. Immuno-virological response and associated factors amongst HIV-1 vertically infected adolescents in Yaoundé-Cameroon. PLoS One. 2017;12(11):1-10. doi:10.1371/journal.pone.0187566

41. Bermudez LG, Ssewamala FM, Neilands TB, et al. Does Economic Strengthening Improve Viral Suppression Among Adolescents Living with HIV? Results From a Cluster Randomized Trial in Uganda. AIDS Behav. 2018;22(11):3763-3772. doi:10.1007/s10461-018-2173-7

42. Rooks, M.G and Garrett, W.S 2016. 峸㽖 HHS Public Access.Physiol Behav. 2017;176(3):139-148. doi:10.1080/09540121.2016.1189496. Disparities in Achieving and Sustaining Viral Suppression 
among a Large Cohort of HIV-Infected Persons in Care Washington, DC Amanda

43. Degefa M, Dubale B, Bayouh F, Ayele B, Zewde Y. Validation of the PHQ-9 depression scale in Ethiopian cancer patients attending the oncology clinic at Tikur Anbessa specialized hospital. BMC Psychiatry. Published online 2020. doi:10.1186/s12888-020-02850-3

44. Logan PGB and GD.

doi:10.1016/j.psychres.2013.07.015. Validity of the Patient Health Questionnaire-9 for Depression Screening and Diagnosis in East Africa

\section{Figures}



Figure 1

Baseline CD4 among cases and controls 